\title{
Programa de Enfermería UNAB: 10 años transformando la enfermería a través del conocimiento
}

\author{
UNAB Nursing Program: Ten years transforming nursing profession \\ through knowledge

\section{Programa de Enfermagem da UNAB: 10 anos transformando a enfermagem através do conhecimento}

Hace 10 años, en enero de 2008, la Universidad Autónoma de Bucaramanga (UNAB) inició la primera cohorte de estudiantes del Programa de Enfermería. No obstante, para hablar sobre qué significan estos 10 años para el Programa de Enfermería, se debe recordar cómo nació la idea de crear la Facultad de Ciencias de la Salud.

La Universidad Autónoma de Bucaramanga inició su recorrido por el área de las Ciencias de la Salud en 1995, cuando se propuso ampliar la orientación de su desarrollo, hasta ese momento en áreas como Ciencias Sociales, Administrativas y en Pedagogía.

Es así como en el año 1996 emprendió labores el Programa de Medicina; desde ese momento se planteó, de manera concurrente, el compromiso de favorecer la interdisciplinariedad con el fin de que los estudiantes pudieran participar del estudio del proceso salud - enfermedad desde las perspectivas de otras profesiones como enfermería, nutrición, terapias, etc.

En 1998, se reactiva la idea de ofrecer una segunda carrera en salud; se piensa en Enfermería, con el objetivo que sus graduados llegaran a marcar la diferencia y dieran respuestas reales a las necesidades de cuidado de enfermería de la región, el país y el mundo. Su estudio de viabilidad fue encargado al Centro de Estudios Estratégicos y en ese momento la Dra. Eulalia García Beltrán, la Dra. Luz Marina Corso Morales, la entonces estudiante de la UNAB Marcela Peralta, hoy directora de Impulsa Unab, y la Enfermera Olga Lucía Gómez Díaz, en ese momento Jefe de la División de enfermería de la FOSCAL, iniciamos a escribir este sueño. En dicha oportunidad, se recomendó no iniciar la puesta en marcha del programa, debido a las condiciones del mercado, a pesar de que la viabilidad académica estaba dada.

En el 2000, la UNAB formula su plan de desarrollo y este recomienda, de manera específica, la creación del programa de Enfermería como una manera de impulsar la naciente Facultad de Ciencias de la Salud y es de esa manera como se aborda nuevamente el tema, procurando explorar otros escenarios que den fuerza a la carrera y la posicionen entre las preferencias de los jóvenes para cursar estudios universitarios. 
En febrero de 2003, se convoca la conformación de un grupo focal para realizar la formulación del proyecto que, en dicho momento, incluyó una propuesta académica, el mejoramiento de la utilización de los escenarios disponibles en la capacidad instalada, tales como laboratorios, aulas y la necesidad de personal tanto docente como administrativo con amplia experiencia en gestión académica en el área de la salud y de Enfermería; por ello se integra al equipo de trabajo la enfermera norteamericana Maureen Balaguera.

Los criterios para la realización del estudio fueron orientados particularmente hacia los aspectos curriculares del programa con base en una valoración y revisión de planes de estudios de instituciones educativas que ofrecían Programas de Enfermería; se tomaron como referencia instituciones tanto del país como fuera de él. Esto generó que en septiembre de 2006 se obtenga el registro calificado para el Programa de Enfermería y se inicie el cumplimiento de ese sueño: lograr formar profesionales de enfermería que marcaran la diferencia y dieran respuestas reales a las necesidades de cuidado de enfermería de la región, el país y el mundo. Finalmente, en enero 14 de 2008 inicia la primera cohorte con 26 estudiantes.

En este inicio del programa nos acompañaron profesores de los programas de Medicina y Psicología como Gloria Arenas Luna, Pedro Suescum Barbosa, Edgar Giovanny Corzo, Vladimir Saldarriaga Tellez, Fernando Mantilla McCorminck, Carolina Beltran Dulcey, Laura del Pilar Cadena Afanador, Rafael Zamora Prieto y, desde el Programa de Enfermería, Maribel Esparza Bohórquez, Marlín Delgado, Senieth Peñaloza, Socorro Fajardo Nates, Pilar Abreu Peralta y Martha Isabel Robles Carreño, con el apoyo permanente de las secretarias del Programa de Medicina Dayra Salcedo y Constanza Ramírez y la asesoría académica y administrativa de la Dra. Liliana Quiñonez Torres Directora del Programa de Psicología y de la Dra. Luz Marina Corso Morales Decana de la Facultad de Ciencias de la Salud.

Durante estos 10 años han pasado muchas cosas que nos han fortalecido en ese sueño de lograr transformar la enfermería a través del conocimiento, con un currículo que permite la interdisciplinariedad y fundamenta la formación integral del Plan Educativo Institucional. En el año 2013 se crea el grupo de investigación CARING y se da inicio al semillero de investigación HIGIA en honor a la diosa de la curación, la limpieza y la sanidad; además, como dinámica institucional de autoevaluación para internacionalización del currículo, se revisan los planes de estudio de universidades como la Universidad de Barcelona, la Universidad de Salamanca y la Universidad de Londres; la Universidad de Nuevo México, el Tecnológico de Monterrey, la Universidad de Buenos Aires y la Pontificia Universidad de República Dominicana; la Universidad de Pennsylvania, la Universidad del Norte de la Florida y la Universidad del Sur de la Florida. Este proceso es acompañado por pares internacionales como la Dra. Sandra Cadena de la Universidad del Sur de la Florida y asesora Fullbright para el proceso de investigación al interior del Programa académico, la asesoría de la Dra. Rosario Cartagena de TC3 para la inclusión del inglés en cursos del componente específico del plan de estudios y la asesoría de la Sra. Ruth Harrold de la Universidad del Norte de la Florida. Esta asesoría internacional dio como resultado que en el segundo semestre de 2010 saliera la primera estudiante del Programa de Enfermería a una pasantía internacional por la Universidad del Sur de la Florida.

El crecimiento de estos diez años no hubiera sido posible sin el apoyo incondicional de la FOSCAL, en particular el Dr. Virgilio Galvis Ramírez y la Enf. Maribel Esparza Bohórquez. Ellos, con inquebrantable voluntad, han permitido que en los escenarios clínicos y administrativos de la FOSCAL y FOSCAL Internacional nuestros estudiantes tengan un escenario particular y sean los preferidos para realizar las prácticas formativas. En este espacio se integra la docencia a la asistencia; allí sus profesionales de enfermería son los profesores de las prácticas formativas de nuestros estudiantes. En adición, contamos con el apoyo de otros escenarios de práctica formativa que en el transcurso de los años nos han acogido como son el Instituto de Salud de Bucaramanga ISABU con su Hospital Local del Norte y sus Centros de Salud, el Instituto del Sistema Nervioso del Oriente ISNOR, el Instituto del Corazón de Bucaramanga, La Fundación Cardio Infantil IC, Hogar del adulto mayor necesitado y el Hospital Psiquiátrico San Camilo, entre otros. Además, se cuenta a partir de este 2018 con un moderno y funcional laboratorio de simulación que nos permite incorporar la innovación como parte fundamental del desarrollo académico y de la capacidad competitiva. Esto genera un logro en la integración horizontal transdisciplinaria y vertical entre las ciencias básicas y las clínicas con integración interdisciplinar, que procura la mejora de las competencias de nuestros estudiantes de pregrado, posgrado y de educación continua. El laboratorio de simulación también facilita el 
cumplimiento de las políticas de educación en salud a través de un entrenamiento académico, que minimiza los riesgos de los pacientes y maximiza la seguridad tanto para el estudiante, el paciente, la institución educativa y el escenario de practica formativa.

Pero este crecimiento no solo ha sido en infraestructura física, escenarios de práctica y dotación. Al cumplir diez años tenemos una población estudiantil de 264 jóvenes en pregrado, 193 graduados de Enfermería y 1473 participantes en programas de extensión solo en los últimos dos años, así como una población de 10 profesores de planta y 30 profesores de cátedra de diferentes áreas del saber. Además, se ha desarrollado el grupo de investigación CARING en donde docentes, jóvenes investigadores y miembros del semillero de investigación adelantan investigaciones colaborativas con otras universidades del país y del mundo; esto hace que se generen publicaciones en revistas nacionales e internacionales. También se cuenta con un medio de difusión de la información científica: la Revista MedUNAB, consolidada como revista y como Escuela Editorial, actualmente liderada por una enfermera, la Dra. Mary Luz Jaimes Valencia.

Nuestra labor interdisciplinar se ha visto resaltada por la participación y apoyo de docentes y estudiantes de los tres programas de la Facultad de Ciencias de la Salud (Enfermería, Medicina y Psicología) en proyectos como el Programa PIES (Programa Interdisciplinar de Educación en Salud) que desarrolla acciones en el Centro de Salud Campo Hermoso, Colegio Aurelio Martínez Mutis, y en los Centros Vida de la Alcaldía de Bucaramanga.

En el proceso de fortalecimiento académico se ingresó a la Asociación Colombiana de Facultades de Enfermería, ACOFAEN; en el año 2013 en transitoriedad y en el año 2017 como programa afiliado. En adición, estamos vinculados con la Red de Enfermería del Oriente Colombiano REOC; allí se trabaja con universidades como la UPTC, Sur Colombiana, Unillanos y Unisangil, la Red de cuidado cultura de la salud, Red Iberoamericana de Líderes en Salud Ambiental Infantil (Red SAMBI), Registred Nurses' Association Ontario (RNAO), Sigma Theta Tau International, Red Colombiana de educación en cuidado paliativo, Red Latinoamericana Cuidado al Paciente Crónico y su Familia, la Red Internacional de Enfermería Quirúrgica (REDIENQUI), la Red de evaluación y promoción de habilidades para la vida y el Grupo Santandereano de Investigación y Asesorías a Personas con Heridas y Ostomías GSIAPHO. A partir de este trabajo conjunto con las ya mencionadas universidades, se desarrollan proyectos en la academia, la extensión y la investigación.

Estos diez años nos ha permitido ser cada día mejores personas y mejores profesionales, pero desde la comodidad de la rutina es muy complicado desafiar nuestros límites. Sin embargo, cuando nos vemos obligados por diferentes circunstancias a salir de la zona de confort por la que nos movemos habitualmente, muchas cualidades y habilidades que no habían tenido la posibilidad de manifestarse lo acaban haciendo, descubriendo así cualidades de nosotros mismos que desconocíamos. Esto es lo que la certificación internacional como Organización Líder de las mejores prácticas académicas BPSO académico (por sus siglas en inglés) nos ha permitido para hacer del mundo un mejor lugar para vivir, innovando en la academia, y ser actualmente el primer Programa de Enfermería certificado como BPSO académico del país y el segundo en Latinoamérica.

Tenemos muchas razones para celebrar y lo estamos haciendo. En este año 2018 el Congreso de la Facultad de Ciencias de la Salud está siendo coordinado por el Programa de Enfermería, junto con Medicina y Psicología, la temática Práctica Basada en la Evidencia. En el caso del programa de Enfermería es una respuesta al sello de calidad que la Certificación Internacional como Centro Comprometido con la Excelencia BPSO (por sus siglas en Ingles Best Practice Spotlight Organization) ha impuesto en nosotros. Queremos ser los mejores para beneficio de nuestros pacientes, nuestros estudiantes, nuestros graduados y nuestra UNAB.

Para el futuro estamos comprometidos con acciones como la acreditación nacional del Programa de Enfermería y la acreditación internacional Arcu-Sur. Esperamos mantener la certificación internacional de la RNAO y convertirnos en un BPSO anfitrión con capacidad para administrar, en nombre de la RNAO, el programa completo de designación como BPSO para un grupo de centros académicos; también buscaremos llegar a la categoría B del grupo de investigación CARING, generar la oferta de postgrados, fortalecer el Centro de Desarrollo en Educación y Tecnología en Salud como el mejor centro de educación continua en salud del país y continuar con los procesos de mejoramiento académico, en investigación y en extensión, para transformar la enfermería a través del conocimiento y llegar a ser uno de los mejores programas de enfermería del país y de Latinoamérica; 
como ya lo había dicho Paulo Freire "la educación no cambia el mundo, cambia a las personas que van a cambiar el mundo".

No quiero terminar sin agradecer a las directivas de la Universidad por haber confiado en nosotros su sueño durante estos 10 años, especialmente al Dr. Alberto Montoya Puyana, a la Dra. Eulalia García Beltrán, al Dr. Gilberto Ramírez Valbuena y a nuestros decanos, la Dra. Luz Marina Corso Morales y nuestro actual decano Dr. Juan José Rey Serrano.

Olga Lucía Gómez Díaz, Enf., Esp., MSc. Directora Programa de Enfermería Universidad Autónoma de Bucaramanga. 